FIV

\section{Sarah M. A. Caney}

The last 30 years have seen advances in our ability to diagnose FIV with some improvements in our ability to manage this disease.

FIV is typically spread by biting and therefore is most common in free-roaming entire male cats. Primary infection (first 2-4 months) is accompanied by transient mild illness, which may go unnoticed, and may include signs such as pyrexia and lymphadenopathy. This is followed by a prolonged asymptomatic period, which often lasts many years. During this period, there is a progressive decline in immune function where the virus particularly targets T-helper lymphocytes (CD4 cells) resulting in immunosuppression.

Clinical signs are extremely variable and nonspecific, including:

- Vague systemic signs such as malaise and weight loss

- Direct viral effects may result in gingivostomatitis,

chronic diarrhoea, proteinuria, neurological and ocular inflammatory lesions

- Immunosuppression may be clinically manifested as increased vulnerability to secondary infections and development of tumours, particularly B cell lymphoma.

Neutropenia, thrombocytopenia and anaemia are the most common haematological abnormalities detected. A polyclonal hyperglobulinaemia is often seen on serum biochemistry.

Diagnosis can often be made by testing for serum or salivary antibodies to FIV envelope or core proteins. A confirmatory test is recommended where possible as false-positive and-negative results are possible. For example, in kittens born to an infected queen, the antibody test should not be used before 6 months of age since maternally derived antibodies acquired through ingesting colostrum may be detected on blood tests. Virus isolation and western blot antibody tests have been traditionally considered the 'gold standard' confirmatory test. The reliability of polymerase chain reaction (PCR) testing depends on the design of the primers used and field strains in the cat's area. More recently quantitative PCR has become available and can help provide information on the magnitude of proviral load.

Management varies according to the stage of disease and clinical signs present.

- Specific therapies - reverse transcriptase inhibitors, such as zidovudine may confer some benefits. Recombinant feline interferon may have some beneficial immunomodulatory effects

- Non-specific support - prompt and aggressive treatment for secondary infections (e.g. bactericidal antibiotics, consider a longer course of treatment) and neoplasia is indicated and can be very successful

- Preventative healthcare - vaccination is generally recommended since FIV-infected cats may be more vulnerable to severe disease associated with infection. Regular check-ups are indicated to monitor health and provide support and advice on general healthcare. Minimizing stress and overcrowding has recently been shown to have an impact on outcome

- FIV-infected cats should be neutered and housed indoor-only if possible. This limits their ability to spread disease to cats outside the home and also reduces their risk of infection from others. The risk of transmission within the household is usually very low (if the cats do not fight) as transmission through mutual grooming, sharing of food bowls etc. is inefficient.

\section{KEY LEARNING OBJECTIVES}

- Describe the clinical presentations associated with feline immunodeficiency virus (FIV)

- List the diagnostic tests available

- Recommend a management plan for an FIV-infected individual

\section{MULTIPLE CHOICE QUESTIONS}

1. Which of the following statements about FIV is FALSE?

(A) A hypergammaglobulinaemia is common on biochemistry

(B) Haematological changes include mild anaemia, neutropenia and lymphopenia

(C) Kittens are commonly infected in utero and via ingestion of infected milk

(D) Up to $20 \%$ of FIV-infected cats do not produce antibodies detectable on patient-side test kits

2. Which of the following diagnostic tests is considered to be 'gold standard' for FIV?

(A) PCR

(B) Salivary gp40 or p24 antibody test

(C) Serum gp40 or p24 antibody test

(D) Virus isolation

3. Which of the following is not recommended following a diagnosis of FIV?

(A) House separately from other cats in the household

(B) Keep indoor only

(C) Neuter

(D) Vaccinate for cat 'flu and enteritis 